\title{
Descripción del contexto hidrogeológico y del patrimonio hidrogeoarqueológico asociado a la Fortaleza de La Mota (Alcalá la Real, provincia de Jaén, sur de España)
}

\author{
Matías Mudarra Martínez(1), Antonio González Ramón ${ }^{(2)}$, Francisco Roldán-García(2),Tomás \\ Peinado Parra ${ }^{(2)}$, Carlos Calvo Aguilar ${ }^{(3)}$, José Luis Hidalgo Cano ${ }^{(3)}$ y Sergio Martos Rosillo ${ }^{(2)}$ \\ (1) Departamento de Geología y Centro de Hidrogeología de la Universidad de Málaga (CEHIUMA), \\ mmudarra@uma.es \\ (2) Oficina de Proyectos del IGME en Granada, Urb. Alcázar del Genil, 4, Edf. Zulema bajo, 18006 Granada, España. \\ antonio.gonzalez@igme.es, s.martos@igme.es, fj.roldan@igme.es \\ (3) Ayuntamiento de Alcalá la Real, Plaza del Ayuntamiento, 1. \\ carlos.calvo@alcalalareal.es, joseluis.hidalgo@alcalalareal.es
}

\begin{abstract}
RESUMEN
La Fortaleza de La Mota es un recinto defensivo levantado en la cúspide del cerro homónimo, en la localidad jienense de Alcalá la Real. Aunque presenta elementos anteriores, se considera que fue durante la época nazarí (siglos XIII y XIV) cuando alcanzó su mayor esplendor. Su ubicación, en el límite entre los reinos cristianos y musulmanes, provocó que esta fortaleza constituyese el principal baluarte defensivo de la frontera norte del antiguo Reino de Granada. Este hecho ocasionó que fuese asediada reiteradamente y que sus habitantes contaran con un sistema de aguada particular, que combinaba el uso del agua subterránea, almacenada en calcarenitas de edadTortoniense, y del agua de lluvia, recolectada en numerosos aljibes distribuidos intramuros. En este trabajo se analiza y se discute el potencial de este particular sistema de abastecimiento. Para ello, se describe el funcionamiento hidrogeológico del pequeño acuífero sobre el que se construyó la fortaleza musulmana, se presenta el sistema de pozos y galerías subterráneas que se ocultaban en su interior, se evalúan sus recursos y sus posibles reservas, y se detallan las características físico-químicas de las aguas subterráneas. Se presta especial atención a la relevancia que pudo tener una de las captaciones más emblemáticas (Pozo de la Conquista) en el sistema de abastecimiento, así como al papel que jugó dicho pozo en la toma de la ciudad por parte de las tropas cristianas de Alfonso XI.
\end{abstract}

Palabras clave: abastecimiento urbano, calidad de aguas, infraestructura hidráulica, patrimonio hidrogeológico, recursos hídricos.

\section{Hydrogeological context and hydrogeoarchaeological heritage associated with the La Mota Fortress (Alcalá la Real, province of Jaén, southern Spain)}

\begin{abstract}
La Mota Fortress is a medieval military enclosure erected on the upper part of the homonymous hill, in Alcalá la Real (province of Jaén, southern Spain). Although previous vestiges have been found, the fortress reached its greatest splendour during the Nasrid-dynasty period (13th and 14th centuries). Its geographical location, in the limit between the old Christian and Muslim kingdoms, conferred a great strategic value to this military complex as a defensive bastion in the northern border of the ancient Kingdom of Granada. This fact caused the citadel to repeatedly be besieged and its inhabitants put into practice a particular system for water supply, based on the combined use of groundwater, stored in the calcareous sandstones of Tortonian age, and rainwater, collected in numerous cisterns sited within city walls. In this paper, the potential interest of this particular supply system is analyzed and discussed. To this end, we describe the hydrogeological functioning of the small aquifer on which the Nasrid fortress was built, and the system of underground wells and galleries that was hidden inside. In addition, the water resources and possible groundwater reserves are evaluated and their physico-chemical characteristics are detailed. Finally, the relevance in the water supply system of one of the most significant withdrawal facilities (Pozo de la Conquista) is analyzed, as well as the role played by the well in the conquest of the city by the Christian troops of Alfonso XI.
\end{abstract}

Keywords: urban water supply, water quality, hydraulic infrastructure, hydrogeological heritage, water resources. 


\section{Introducción}

De entre los muchos sistemas de abastecimiento basados en el uso del agua subterránea en la época de Al-Ándalus, debe ser destacado el existente en la Fortaleza de La Mota (antigua ciudad amurallada de Alcalá), por haber sido el centro de relevantes hechos históricos, acontecidos en la Baja Edad Media. Esta fortaleza, con una superficie intramuros de 2,7 ha, se encuentra en la localidad jienense de Alcalá la Real, en el sur de España (Figs. 1 y 2a). Se trata de un asentamiento fortificado sobre una pequeña meseta elevada que constituye un emplazamiento geoestratégico privilegiado, ya que permitía el control de amplias extensiones de los terrenos circundantes y que, además, era fácil de proteger ante los asedios de las tropas enemigas (Calvo-Aguilar, 2017; Martos-Rosillo et al., 2018).

Esta antigua alcazaba andalusí, enclavada sobre una mesa coronada por calcarenitas del Mioceno superior, sufrió un largo asedio por parte de las tropas del rey Alfonso XI de Castilla, en su avance hacia la capital del Reino de Granada (Calvo-Aguilar, 2017). EI ejército musulmán pudo resistir el duro asedio gracias

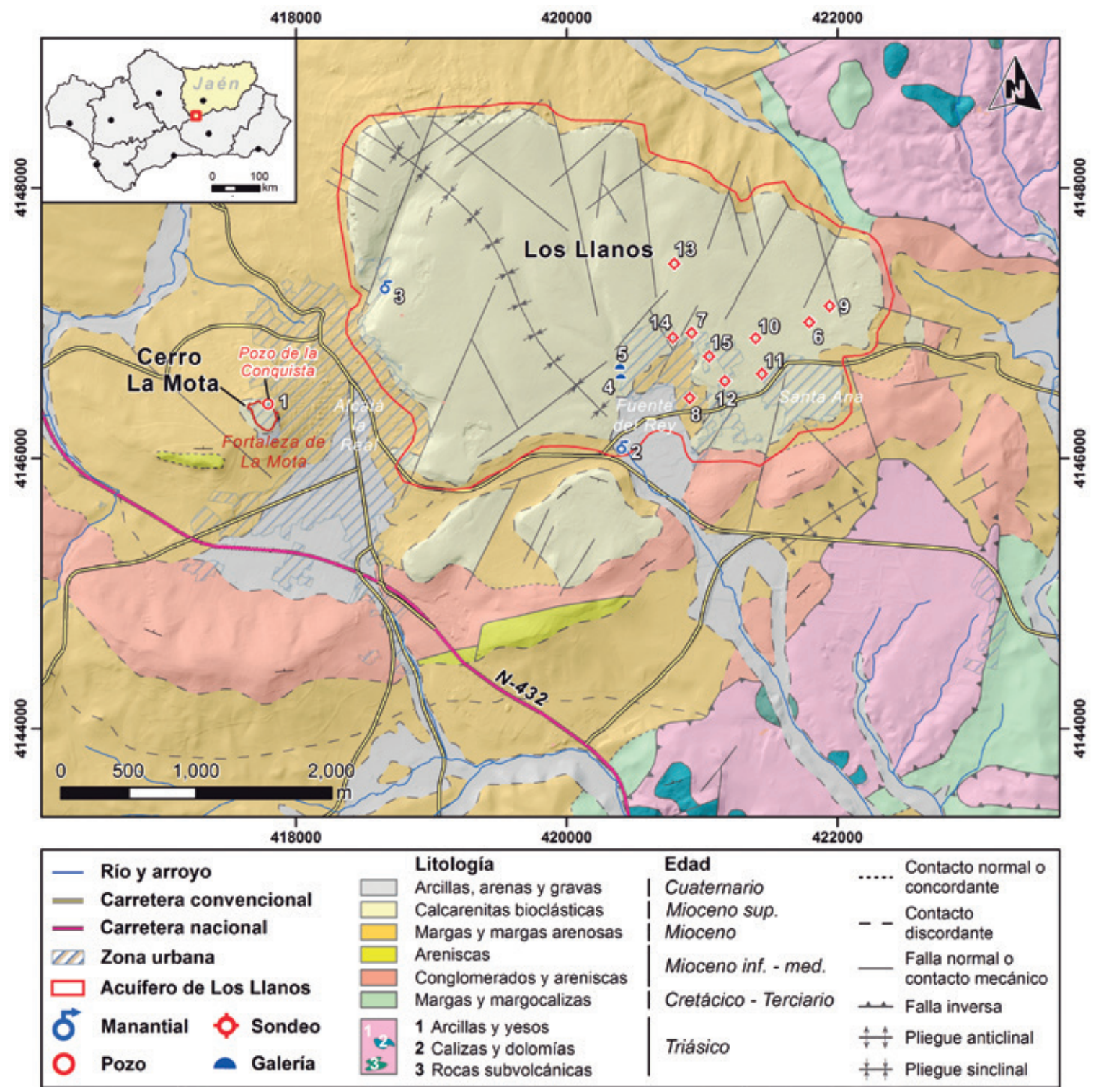

Figura 1. Localización geográfica y contexto geológico del entorno de Alcalá la Real y del acuífero calcarenítico mioceno de Los Llanos. Cartografía geológica tomada de Díaz de Neira et al. (1991). La numeración se corresponde con la indicada en la Tabla 1.

Figure 1. Geographic location and geological context of Alcalá la Real and the surrounding areas, where the Los Llanos aquifer (calcareous sandstones of Miocene age) is located. Geological mapping taken from Díaz de Neira et al. (1991). The numbering corresponds to that indicated in Table 1. 


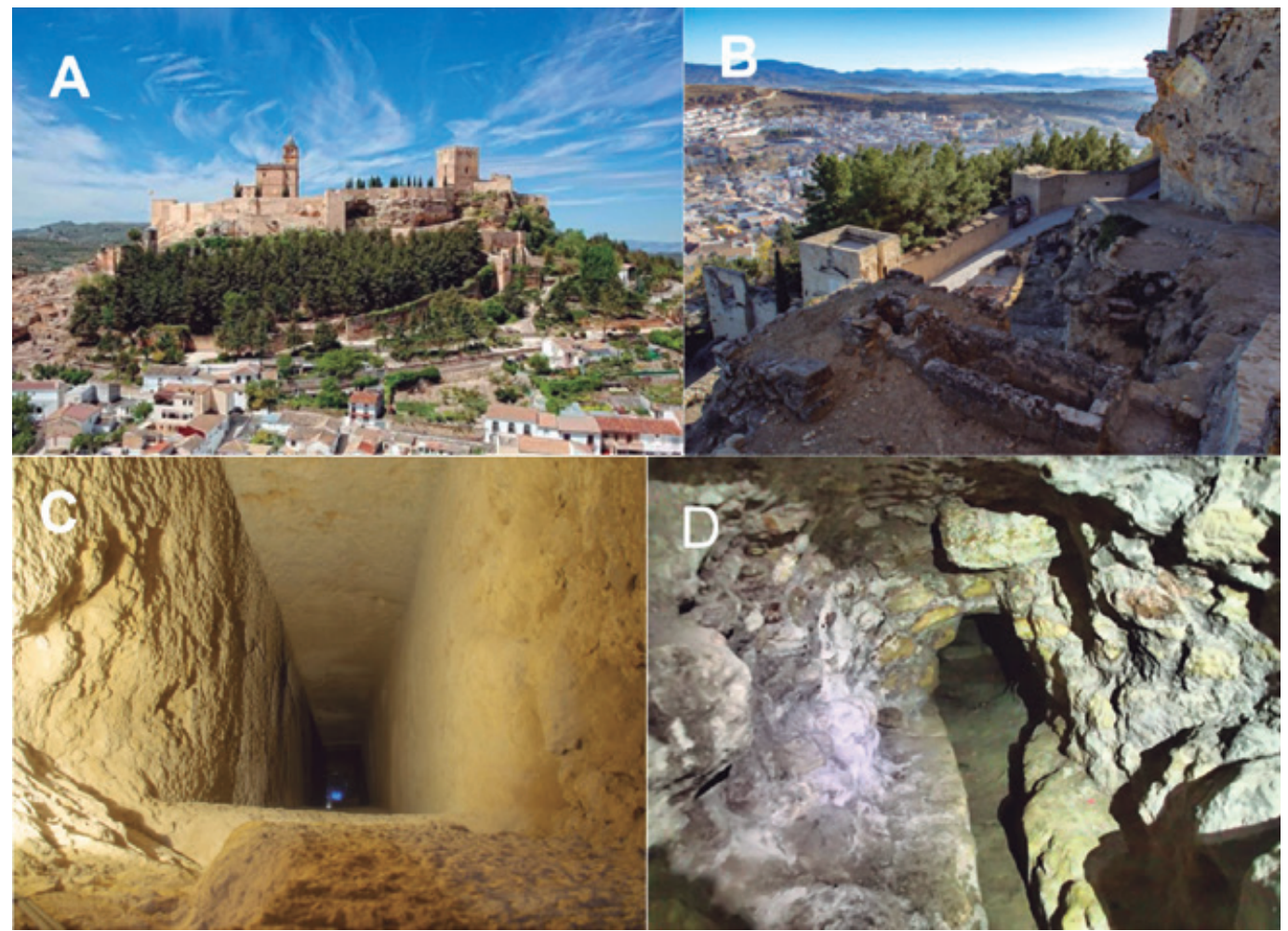

Figura 2. A, Panorámica de la Fortaleza de La Mota desde el Este. B, Brocal del Pozo de la Conquista, localizado en el interior de la zona amurallada. C, Vista interior del Pozo de la Conquista desde la galería de acceso. D, Galería construida en la base de la Torre de la Cárcel Real, en el límite sur del perímetro amurallado.

Figure 2. A, Overview of La Mota Fortress from the east. B, Pozo de la Conquista parapet, located inside the citadel. $C$, View from the access gallery of the Pozo de la Conquista interior. D, Gallery built at the base of the Torre de la Carcel Real, on the southern edge of the military enclosure.

a la existencia de varios pozos excavados en las calcarenitas y al elevado número de aljibes donde se recolectaba el agua de lluvia. El pozo más emblemático, conocido actualmente como Pozo de la Conquista, se encuentra situado extramuros de la Fortaleza, bajo el suelo de un arrabal contiguo a la alcazaba, y protegido por laTorre del Pozo (Figs. 2b y c). Durante el asedio de las tropas cristianas, los musulmanes accedían a esta captación mediante una galería subterránea, o mina de aguada, que desciende desde el patio de la alcazaba hasta el brocal subterráneo del pozo. Según se indica en la Crónica de Alfonso Xl, un andalusí apresado durante el asedio reveló la ubicación del pozo a cambio de su libertad. Conocida la situación del pozo, las tropas cristianas excavaron varias galerías hasta dar con él, impidiendo la aguada de la alcazaba y contribuyendo esta vía de acceso al recinto a la toma de la Fortaleza, en el año 1341. Fue así como cayó uno de los últimos bastiones de la frontera norte del Reino de Granada (Calvo-Aguilar, 2004, 2017).

En este trabajo se han evaluado los recursos medios renovables que pueden recargar el pequeño acuífero del cerro de La Mota, su capacidad de almace- namiento y las características hidrogeoquímicas del agua subterránea; asimismo, se describe el modelo conceptual de su funcionamiento. Finalmente, se discute la incidencia que pudo tener en la toma de la antigua alcazaba la inutilización del denominado Pozo de la Conquista.

\section{Contexto geológico}

La secuencia sedimentaria de la cuenca de Alcalá la Real comienza con calizas, conglomerados, margas y margo-calizas de edad Mioceno inferior, sobre las que se disponen conglomerados, areniscas, calizas con algas y margas del Mioceno medio. Estos sedimentos se extienden al sur de la localidad de Alcalá la Real y de la pedanía de Santa Ana (Fig. 1). Sobre los materiales anteriores, se dispone una alternancia de margas blancas con delgadas intercalaciones de calcarenitas que afloran en el sector de los Arrabales de la Fortaleza de La Mota (Fig. 3). La secuencia culmina con calcarenitas bioclásticas, de edad Tortoniense superior, sobre las que se asienta la Fortaleza de La Mota, y que presentan vistosas estructuras de ordenamiento inter- 


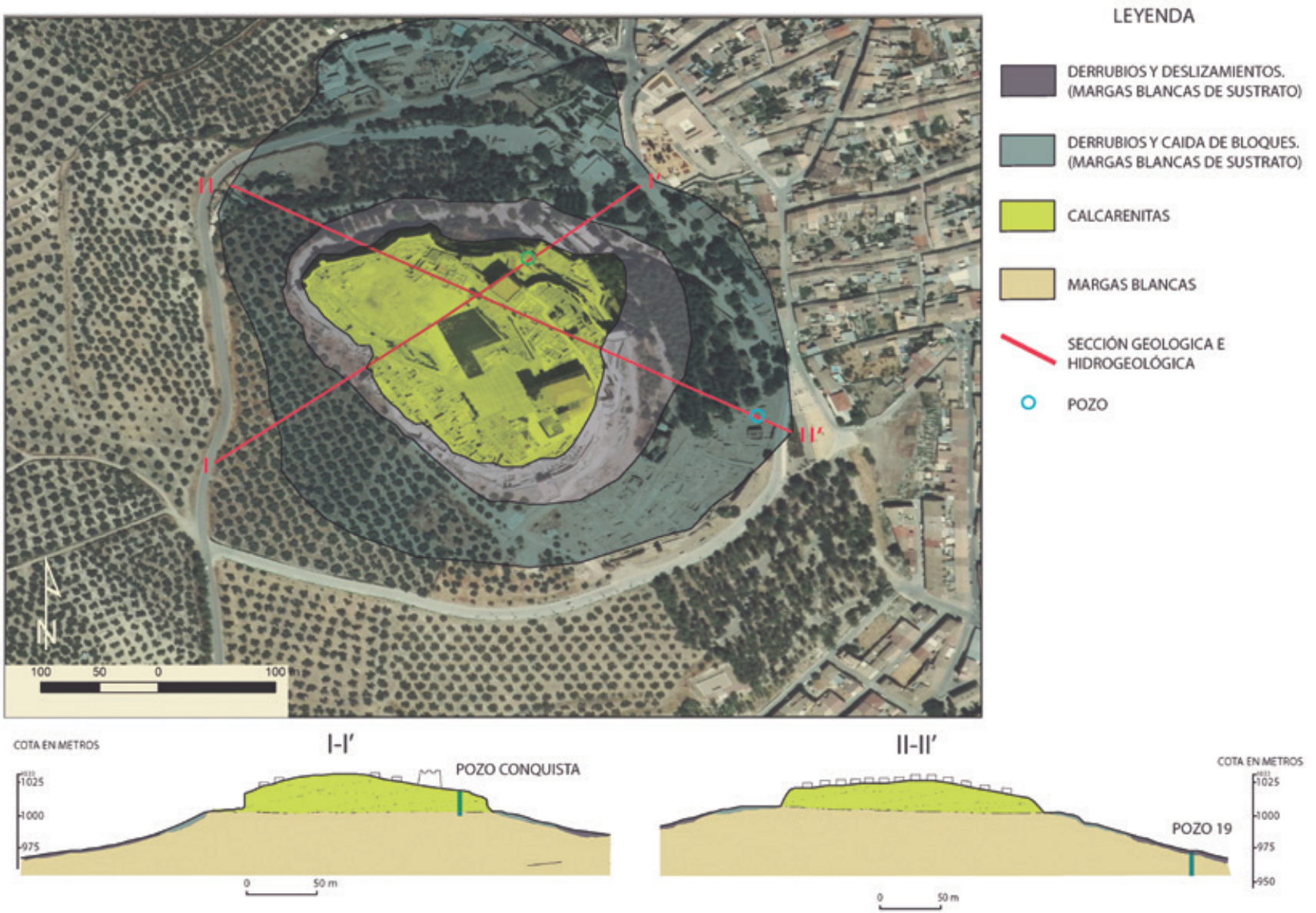

Figura 3. Esquema geológico y secciones del cerro de la Mota y de su entorno inmediato.

Figure 3. Geological sketch and geological cross sections of the cerro de La Mota and the surrounding area.

no tipo estratificaciones cruzadas. Estos materiales corresponden a la última fase del relleno sedimentario (transgresión del Mioceno superior) de la cuenca de Alcalá la Real (Rodríguez-Fernández, 1982), que guarda relación con la cuenca del Guadalquivir y la de Granada. Se calcula un espesor del orden de $300 \mathrm{~m}$ para las margas blancas y de un máximo de $100 \mathrm{~m}$ para las calcarenitas (Gollonet et al., 2002; González Ramón et al., 2009; Martín Montañés et al., 2018).

EI Mioceno de esta región muestra una disposición subcircular en cartografía, de manera que los sedimentos más modernos se sitúan hacia el $\mathrm{N}$, superponiéndose de manera discordante al sustrato subbético compuesto por materiales de edades Triásico, Jurásico, Cretácico y Paleógeno. La estructura regional del Mioceno es de una sinforma abierta hacia el $\mathrm{N}$, en la que los sedimentos del Mioceno superior culminan con una estructura subtabular con ligera inclinación al S (Fig. 1). La mayor resistencia a la erosión y disposición geométrica de las calcarenitas ha favorecido el desarrollo de relieves tabulares, como es el caso de la mesa de Los Llanos (Fig. 1). La estructura previa fue afectada por una etapa de fracturación más reciente en la que se produjeron fallas normales según direcciones NE-SO y NO-SE (Roldán et al., 2012; Azañón et al., 2012). La fracturación condiciona la circulación del agua en el interior de las calcarenitas y mejora las propiedades hidráulicas de estos materiales.

\section{Caracterización hidrogeológica}

\section{Descripción geométrica y características hidráulicas de los materiales acuíferos}

Las calcarenitas de edad Tortoniense superior, dispuestas de manera tabular o buzando ligeramente hacia el suroeste, y que afloran al este de Alcalá la Real, constituyen el denominado acuífero de Los Llanos (Fig. 1). Este tiene una extensión de $6,6 \mathrm{~km}^{2}$ de materiales permeables dispuestos sobre la for- 
mación margosa del Mioceno superior que actúa como sustrato de baja permeabilidad. Los recursos hídricos medios renovables de este acuífero han sido evaluados en 1,3 hm³/año (IGME, 1998; González-Ramón et al., 2009), en buena parte utilizados para el abastecimiento de la localidad. Las características hidrogeológicas de estos materiales son excelentes, debido a su elevada porosidad y a su alta permeabilidad (por los procesos de fracturación y karstificación), y a la calidad del agua subterránea, que suele ser apropiada para todo tipo de usos. En este sentido, se debe indicar que los parámetros hidráulicos de la formación permeable del Mioceno superior son bien conocidos gracias a los trabajos realizados por el IGME y por el Ayuntamiento de Alcalá la Real (Gollonet et al., 2002). La transmisividad calculada alcanza valores comprendidos entre 500 y $2.400 \mathrm{~m}^{2} /$ día, lo que hace posible la explotación de caudales comprendidos entre 5 y $24 \mathrm{l} / \mathrm{s}$, con descensos dinámicos de un metro. El coeficiente de almacenamiento calculado mediante ensayos de bombeo es de $2 \times 10^{-4}$, en los sectores en los que está confinado, y la porosidad eficaz media del 2,3\% (IGME, 1998). Las propiedades hidráulicas de las calcarenitas no son, por tanto, un factor limitante para su explotación.

Al oeste del acuífero de Los Llanos se encuentra el cerro de La Mota, consistente en un pequeño afloramiento de la misma formación acuífera, aislado por erosión, de tan solo $0,06 \mathrm{~km}^{2}$ de afloramientos permeables (Figs. 1 y 3). El espesor medio de los materiales permeables en este cerro es de $40 \mathrm{~m}$ y la morfología en planta del afloramiento calcáreo es más o menos circular, a modo de mesa, ligeramente inclinado hacia el sureste y colgado sobre las margas infrayacentes. Esta disposición hace que el desmantelamiento del pequeño cerro testigo se produzca por caídas de rocas que van desprendiéndose y deslizándose por las laderas a favor de las pendientes. Ello da lugar a escarpes verticales que fueron aprovechados para la defensa de la ciudadela fortificada en el pasado. El pequeño acuífero del cerro de La Mota está constituido, por tanto, por $40 \mathrm{~m}$ de calcarenitas y por los derrubios de vertiente que recubren sus laderas y que se disponen perimetralmente a la mesa calcarenítica (Fig. 3). Las propiedades hidráulicas de estos materiales serían las mismas que las del acuífero de Los Llanos; es decir, presentan altos valores de permeabilidad y de porosidad.

\section{Inventario de puntos de agua y de aljibes}

En la Fortaleza de La Mota existen un total de 22 pozos excavados. La mayoría presentan secciones cir- culares con diámetros comprendidos entre 0,75 y 1,5 $\mathrm{m}$ y profundidades que varían entre menos de $1 \mathrm{~m}$ y más de $20 \mathrm{~m}$. Del total de pozos inventariados, $17 \mathrm{se}$ encuentran sobre derrubios de ladera y margas blancas arenosas y otros cinco en las calcarenitas miocenas acuíferas (Fig. 4). Tres pozos se encuentran rellenos casi en su totalidad de derrubios, otros seis están diseñados para almacenamiento de agua de escorrentía y tan solo cuatro alcanzan el nivel saturado. El resto de pozos se encuentran secos actualmente.

Los pozos que captan derrubios y margas se encuentran en su mayoría al sureste del cerro de La Mota, en el antiguo arrabal contiguo a la Fortaleza. Se trata de pozos-almacén, poco productivos, dada la baja permeabilidad de las margas, por lo que serían explotados por el día, para el suministro de una casa, y recuperarían lentamente el nivel estático a lo largo de la noche.

De los cinco pozos excavados directamente sobre las calcarenitas, el más destacado es, sin duda, el Pozo de la Conquista (indicado con el $n^{\circ} 22$ en la figura 4). Este pozo se encuentra en el interior de una torre de $6,5 \mathrm{~m}$ de lado por $10 \mathrm{~m}$ de altura, construida con hiladas de mampuestos unidos con mortero de cal y arena (Fig. 2b). La captación fue excavada en las calcarenitas que afloran al pie de la torre, con una sección rectangular de $2 \times 3 \mathrm{~m}$ y $20 \mathrm{~m}$ de profundidad (Fig. 2c). En la actualidad, el nivel piezométrico se encuentra a $17 \mathrm{~m}$ de profundidad, medidos desde el acceso interior por la galería contigua al pozo (Calvo-Aguilar, 2017).

La elevada transmisividad de las calcarenitas permitiría la rápida recuperación del nivel dinámico después de la extracción de agua; es decir, los pozos que tuviesen un espesor saturado suficiente permitirían un caudal de explotación mayor que el potencialmente extraíble en aquellos tiempos, mediante su explotación con los sistemas de elevación disponibles: cigüeñales, norias de sangre, o simplemente cubos suspendidos con una polea. Sin embargo, el principal problema para la captación de agua subterránea desde el Pozo de la Conquista reside en el escaso espesor saturado de las calcarenitas. Por su ubicación, en el borde norte de los afloramientos permeables, es probable que el pozo solo tuviese algún metro de agua en calcarenitas durante las épocas lluviosas y que el nivel descendiese de forma rápida durante los estiajes, hasta quedarse prácticamente sin columna de agua a la altura de las calcarenitas en los estiajes más prolongados.

Por otro lado, uno de los puntos de agua inventariados en el interior de la Torre de la Cárcel Real, situada en la muralla sur de la Fortaleza, no debe considerarse como un pozo, sino más bien una pe- 


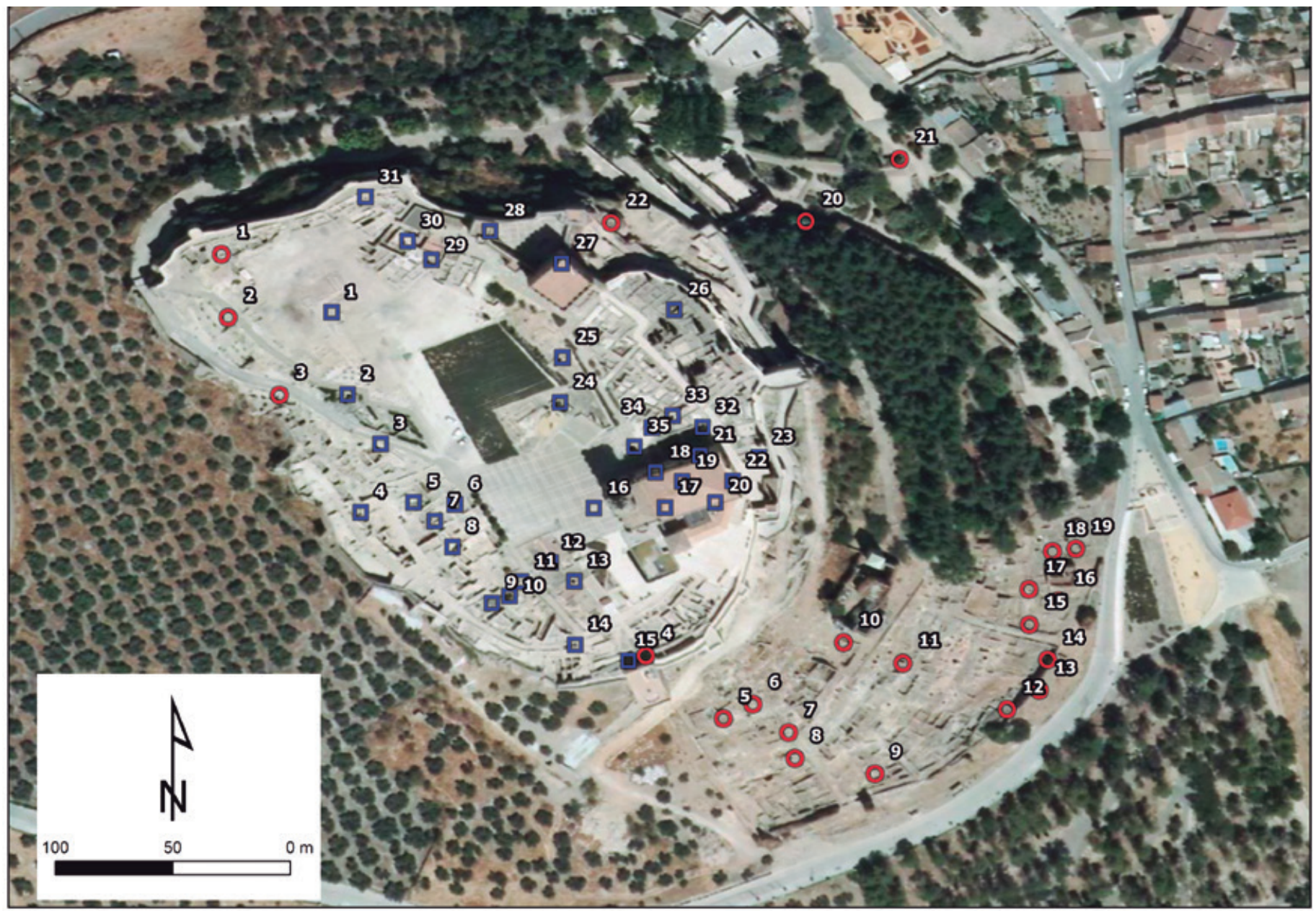

Figura 4. Localización de los pozos (círculos rojos) y aljibes (rectángulos azules) inventariados en el cerro de La Mota. El número 22 corresponde al Pozo de la Conquista ( $n^{\circ} 1$ en figura 1). Inventario de puntos de agua tomado de los trabajos arqueológicos realizados por el Ayuntamiento de Alcalá la Real.

Figure 4. Location of wells (red circles) and cisterns (blue rectangles) inventoried in the cerro de La Mota. The number 22 corresponds to the Pozo de la Conquista ( $n^{\circ} 1$ in Figure 1). Water inventory taken from the archaeological works carried out by the city of Alcalá la Real.

queña galería ubicada en el sótano de la citada torre, en un aljibe subterráneo (Fig. 2d). Por su cota $(\approx 1005$ m s.n.m.), pudiera haber estado operativa durante los años con abundantes precipitaciones.

Por último, debe de ser destacada la presencia de 34 aljibes en el interior de la Fortaleza. Las excavaciones arqueológicas llevadas a cabo han permitido determinar que algunos de estos aljibes datan de la Edad del Bronce y otros del periodo romano, si bien, la gran mayoría se construyeron en la Edad Media (Calvo-Aguilar, 2004). Hay que señalar que estos aljibes fueron utilizados hasta que comenzó el abandono de la Fortaleza a partir del siglo XVIII, y empleados con posterioridad probablemente como osarios y como vertederos hasta su reciente rehabilitación.

\section{Evaluación de recursos y reservas}

EI IGME (1998) realizó una evaluación de la recarga media del acuífero de Los Llanos mediante un balance mensual de agua en el suelo y un análisis de las salidas y de la variación de almacenamiento en el acuífero para los periodos 1984/1999 y 1991/1999. Se utilizaron distintos valores de reserva útil de agua en el suelo y de umbral de escorrentía hasta conseguir ajustar las salidas del sistema más la correspondiente variación de almacenamiento. Con los valores de reserva útil y de umbral de escorrentía calibrados, más los datos meteorológicos de la estación de Alcalá la Real (5406 -AEMET-) correspondientes al periodo 1975-1999, se efectuó un nuevo balance de agua 
en el suelo. Los resultados alcanzados en esta nueva aproximación permitieron obtener valores de recarga

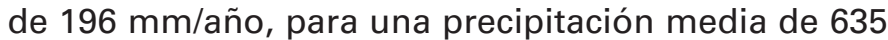
$\mathrm{mm} / \mathrm{año}$. Estos valores implican una tasa de recarga media del $31 \%$, que es perfectamente asumible para el tipo de acuífero y para el contexto regional en el que se encuentra (Martos-Rosillo et al., 2015). Con estos datos, la recarga media del acuífero de Los Llanos $(6,6$ $\mathrm{km}^{2}$ ) resulta de $1,3 \mathrm{hm}^{3} / a$ ño y la del pequeño acuífero del cerro de La Mota $\left(0,06 \mathrm{~km}^{2}\right)$ de 0,012 hmªño.

Las reservas de agua que podían ser explotadas con los pozos existentes en las calcarenitas serían muy limitadas. A modo de aproximación, con un espesor saturado comprendido entre 1 y $2 \mathrm{~m}$ en esta formación, considerando que la lámina de agua se extendiese por todo el sistema $\left(60.000 \mathrm{~m}^{2}\right)$, y con una porosidad del $2 \%$ resultarían unas reservas de agua comprendidas entre 1.200 y $2.400 \mathrm{~m}^{3}$.

\section{Caracterización hidrogeoquímica del agua subterránea}

En la tabla 1 se presentan los valores de los parámetros físico-químicos y de los componentes químicos mayoritarios medidos en las aguas subterráneas del

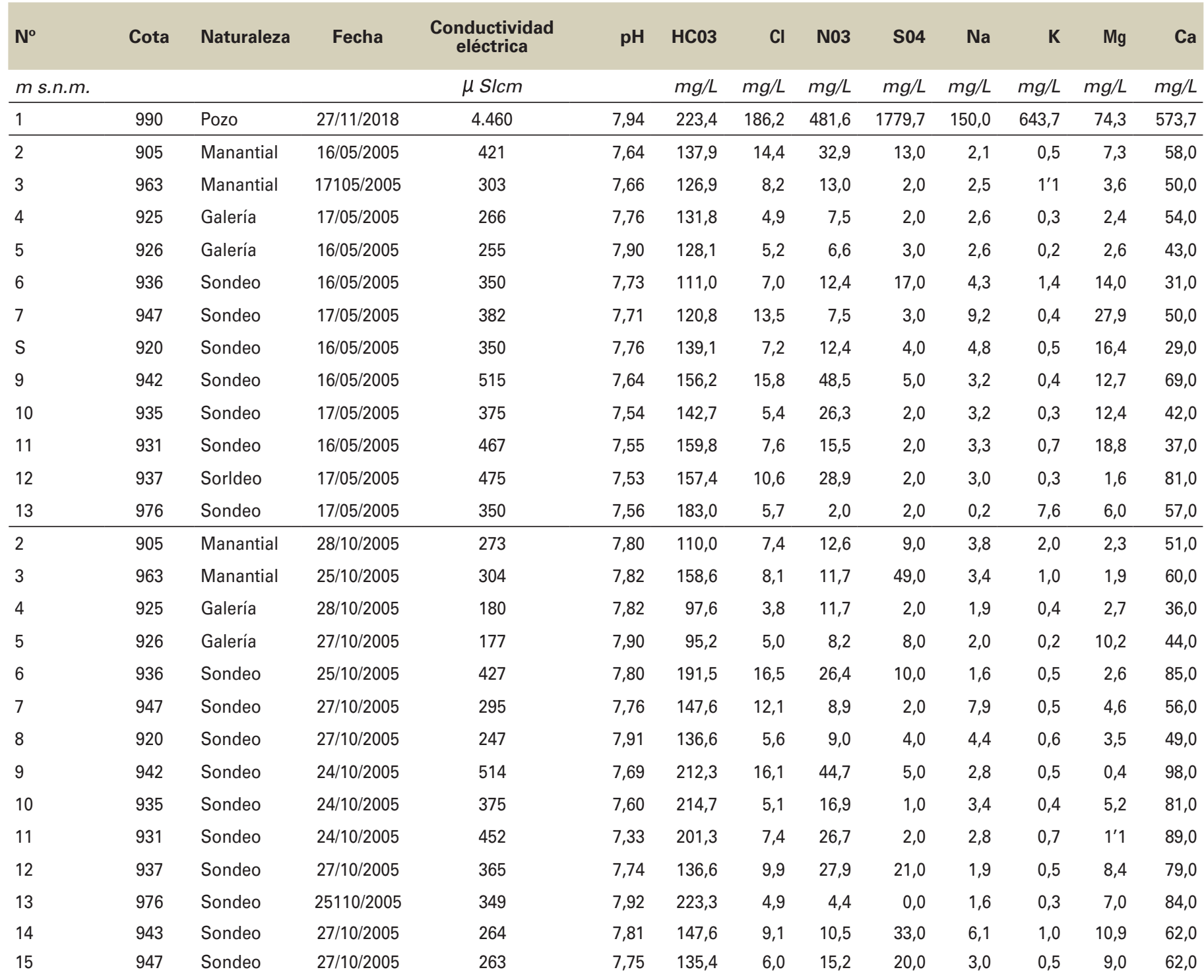

Tabla 1. Valores de las variables físico-químicas y componentes químicos mayoritarios analizados en las aguas subterráneas del acuífero de Los Llanos y del Pozo de la Conquista. Los números corresponden con los puntos indicados en la figura 1.

Table 1. Values of the physico-chemical parameters and major chemical components analyzed in the groundwater of Los Llanos aquifer and from the Pozo de la Conquista. The numbering corresponds to that indicated in Figure 1. 
acuífero de Los Llanos y del vecino cerro de La Mota (Pozo de la Conquista, $\mathrm{n}^{\circ} 1$ en la tabla 1). Los datos procedentes del primero corresponden a las medidas efectuadas en 2005, durante dos campañas diferentes (en aguas bajas-mayo 2005-y altas-octubre 2005-), en los puntos más significativos (manantiales y sondeos, Fig. 1). Los valores medios de conductividad eléctrica del agua del acuífero de Los Llanos están comprendidos entre $177 \mu \mathrm{S} / \mathrm{cm}$ (Fuente Somera) y $515 \mu \mathrm{S} / \mathrm{cm}$ (sondeo $\mathrm{n}^{\circ} 10$ ), aunque la mayoría de las medidas varían entre 300 y $400 \mu \mathrm{S} / \mathrm{cm}$. En casi todos los puntos considerados se registraron valores más bajos de mineralización en situaciones de aguas altas. Estos resultados contrastan con los obtenidos en el Pozo de la Conquista, cuyas aguas presentaban en noviembre de 2018 un valor de conductividad eléctrica significativamente superior $(4.460 \mu \mathrm{S} / \mathrm{cm})$. Ello es consecuencia de los contenidos más elevados en casi todos los componentes hidroquímicos medidos en sus aguas (Tab. 1). Tan sólo la medida de alcalinidad $(223,4 \mathrm{mg} / \mathrm{L})$ es similar a las efectuadas previamente en los diferentes puntos de control del acuífero de Los Llanos.

Las diferencias hidroquímicas detectadas entre las aguas del acuífero de Los Llanos, por un lado, y las del Pozo de la Conquista, por otro, se sintetizan

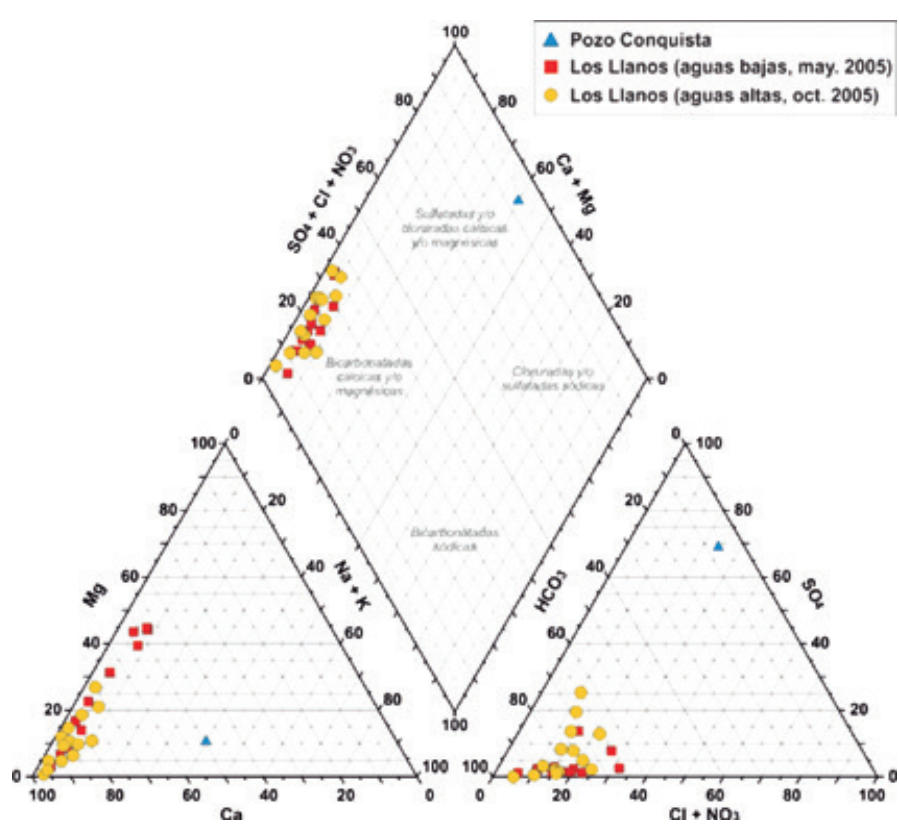

Figura 5. Diagrama de Piper de las muestras de agua subterránea recogidas en los principales manantiales y sondeos del acuífero de Los Llanos y en el Pozo de la Conquista.

Figure 5. Piper diagram of groundwater samples collected in the main springs and wells from Los Llanos aquifer and from the Pozo de la Conquista. en el diagrama de Piper de la figura 5. Con independencia de las condiciones hidroclimáticas en las que tuvo lugar el muestreo, las aguas subterráneas procedentes del primero son de facies bicarbonatada cálcica y bicarbonatada cálcico-magnésica (Fig. 5), dada la naturaleza calcárea de los materiales detríticos acuíferos. Por su parte, el agua del Pozo de la Conquista presenta facies mixtas, del tipo sulfatada cálcico-sódica.

En general, la alcalinidad y los contenidos en $\mathrm{Ca}^{2+}$ y $\mathrm{Mg}^{2+}$ proceden de la disolución de los minerales (calcita y dolomita) que constituyen las formaciones acuíferas presentes en Los Llanos y en el cerro de La Mota. Los altos valores de sulfatos en el pozo $(1.780 \mathrm{mg} / \mathrm{L})$ se pueden relacionar con la lixiviación de los abundantes vertidos que se han ido produciendo y acumulando durante los últimos siglos en el afloramiento calcáreo del cerro de La Mota, y con la disolución de los yesos dispersos entre las margas infrayacentes, que se suponen son captadas en los últimos metros de profundidad del pozo. Parte de los contenidos en $\mathrm{Ca}^{2+}$ y $\mathrm{Mg}^{2+}$ procederían igualmente de la disolución de estos minerales evaporíticos.

Las margas blancas del Mioceno superior sobre las que se apoya la formación acuífera podrían alojar igualmente trazas de halita, cuya disolución proporcionaría parte de los contenidos en $\mathrm{Cl}^{-}$y $\mathrm{Na}^{+}$medidos en las aguas subterráneas del área de estudio (Tab. 1). Es conocido que las aguas contenidas en las margas miocenas marinas de relleno de la depresión del Guadalquivir presentan generalmente elevados valores en cloruros $y$, en menor medida, en sulfatos, que son consecuencias del recuerdo de las aguas marinas miocenas que aún no han sido lavadas por completo (Núñez et al., 2006). La reconcentración en el suelo por evaporación del agua de lluvia (Schoeller, 1962) podría ser otra de las causas probables que explicaría la presencia de ambos iones en el agua. Sin embargo, los valores de $\mathrm{Cl}^{-}$y $\mathrm{Na}^{+}$en el agua del Pozo de la Conquista $(186,2$ y 150,0 mg/L, respectivamente), marcadamente superiores a los registrados en las aguas del acuífero de Los Llanos $(8,6 \mathrm{y}$ $3,4 \mathrm{mg} / \mathrm{L}$ ), no son compatibles con la concurrencia de los dos procesos naturales mencionados (disolución y evaporación); es necesario invocar la existencia de una fuente adicional de halita o bien un mecanismo que favorezca la reconcentración de los iones $\mathrm{Cl}^{-}$y $\mathrm{Na}^{+}$en el agua del Pozo de la Conquista, o ambos factores de manera simultánea. En este sentido, hay que indicar que los altos valores de $\mathrm{K}^{+}(643,7 \mathrm{mg} / \mathrm{L})$ y de $\mathrm{NO}_{3}^{-}(481,6 \mathrm{mg} / \mathrm{L})$ en el agua del pozo refuerza el posible origen antrópico de la alta mineralización del agua del pozo. Estos valores contrastan con las concentraciones medias de ambos parámetros me- 
didos en el agua subterránea del vecino acuífero de Los Llanos $(0,9$ y $17,2 \mathrm{mg} / \mathrm{L}$, respectivamente), los cuales serían más propios de procesos naturales de enriquecimiento en el suelo $\left(<7 \mathrm{mg} / \mathrm{L}\right.$ de $\mathrm{NO}_{3}$, $\mathrm{Mu}$ darra et al., 2014), sin descartar la existencia de una incipiente contaminación agrícola que afectaría al conjunto del acuífero.

El uso, hasta mediados del siglo pasado, de gran parte de la meseta del cerro de La Mota como cementerio, unido al vertido incontrolado de escombros hasta mediados de los ochenta del mismo siglo, y la utilización de numerosos aljibes como osarios y como vertederos improvisados son, muy probablemente, las causas de la contaminación del agua almacenada en dicho pozo. A lo anterior habría que añadir la ocupación de las galerías de acceso al Pozo de la Conquista como cuadras de ganado hasta hace pocos años.

\section{Modelo conceptual de funcionamiento hidrogeológi- co del cerro de La Mota. Relevancia real del Pozo de la Conquista como punto de abastecimiento}

El cerro de La Mota constituye un pequeño acuífero carbonático $\left(0,06 \mathrm{~km}^{2}\right.$ de afloramientos permeables), libre y compuesto por calcarenitas que disponen de un espesor medio de $40 \mathrm{~m}$. Se trata de un acuífero de doble permeabilidad, colgado sobre margas, que se recarga exclusivamente por la infiltración del agua de Iluvia. El carácter abierto al flujo subterráneo de todo el contorno facilita su rápido drenaje, si bien, este flujo se dirige, preferentemente, hacia el punto de menor cota del muro del acuífero, ubicado al sureste del cerro. Hacia este sector se drenan los aproximadamente $12.000 \mathrm{~m}^{3}$ de recursos que se recargan anualmente, de media. La descarga del acuífero satura las margas de matriz arenosa y los bloques de arenisca, embebidos en éstas, que afloran en la zona donde se ubicaba el antiguo arrabal contiguo a la Fortaleza. Ello posibilitó la existencia de pozos poco productivos, dada la escasa permeabilidad de la formación margosa.

La geometría del afloramiento de calcarenitas que da lugar al acuífero del cerro de La Mota, plano y ligeramente basculado hacia el sureste, y el hecho de tratarse de un acuífero colgado sobre las margas condicionan la existencia de una zona saturada con muy escasa capacidad de almacenamiento (Fig. 6). Por este motivo, las reservas que corresponden de 1 a $2 \mathrm{~m}$ de espesor saturado de agua en las calcarenitas implican valores de almacenamiento de 1.200 y de $2.400 \mathrm{~m}^{3}$, respectivamente. Hay que indicar que $2.400 \mathrm{~m}^{3}$ /año corresponden al consumo actual de 26 personas en un año, considerando una dotación de 250 litros/habitante/día. Las dotaciones en la Edad Media serían del orden de 20 litros/habitante/día, por lo que $2.400 \mathrm{~m}^{3}$ permitirían el abastecimiento de algo más de 300 personas durante un año.

Muy probablemente, en los tiempos de asedio de esta Fortaleza, las viviendas, las construcciones y el tránsito de personas en el interior de la zona amurallada contribuirían a reducir la superficie de la zona de recarga efectiva y a reducir la permeabilidad del suelo, con lo que la cifra de recursos antes indicada sería aún menor. Además, la mayor parte del agua recargada en el acuífero no podía ser captada por los habitantes de la ciudadela, dado que una vez infiltrada el agua, ésta se dirigiría hacia la zona sureste del cerro, donde se drenaba de forma lateral hacia las margas miocenas.

En consonancia con lo anteriormente expuesto, el emplazamiento del Pozo de la Conquista, próximo al borde nororiental del afloramiento calcáreo, no es el ideal para captar los recursos de este pequeño acuífero (Fig. 6). En ese sector el espesor de las calcarenitas es menor y la recarga que se produce en esta zona del acuífero se dirige hacia el sureste, una vez alcanzada la zona saturada. Además, es probable que en sus últimos metros la captación alcance o esté cerca de cortar el muro de las calcarenitas (se recuerda que el pozo tiene $20 \mathrm{~m}$ de profundidad, excavados en la roca). En esa situación, el rendimiento hidráulico del pozo sería muy reducido. La ausencia de una plataforma más grande en la parte alta del pozo indica que el agua solo podía ser elevada mediante cubos. El espacio existente impide la instalación de una noria de sangre o de un cigüeñal, que eran los sistemas de elevación de agua desde un pozo disponibles en aquella época. Este hecho vendría a avalar la escasa productividad hidráulica del pozo.

Todo ello lleva a pensar que el Pozo de la Conquista se comporta como un pozo "almacén", con un nivel piezométrico relacionado con los materiales margosos infrayacentes más que con las propias calcarenitas del Tortoniense (Fig. 6), lo que le otorgaría limitada capacidad de renovación del agua en condiciones naturales. Ello contribuiría al estancamiento del agua, a la paulatina degradación de su calidad, y al consecuente enriquecimiento en todas las especies hidroquímicas (Tab. 1; Fig. 5). En cualquier caso, las características hidrogeoquímicas del acuífero del cerro de La Mota no se pueden dilucidar con el muestreo realizado en este trabajo, dado que el agua captada en el Pozo de la Conquista presenta evidentes signos de contaminación, además de corresponder, más bien a agua mezclada con el agua almacenada en las margas arenosas que definen el 


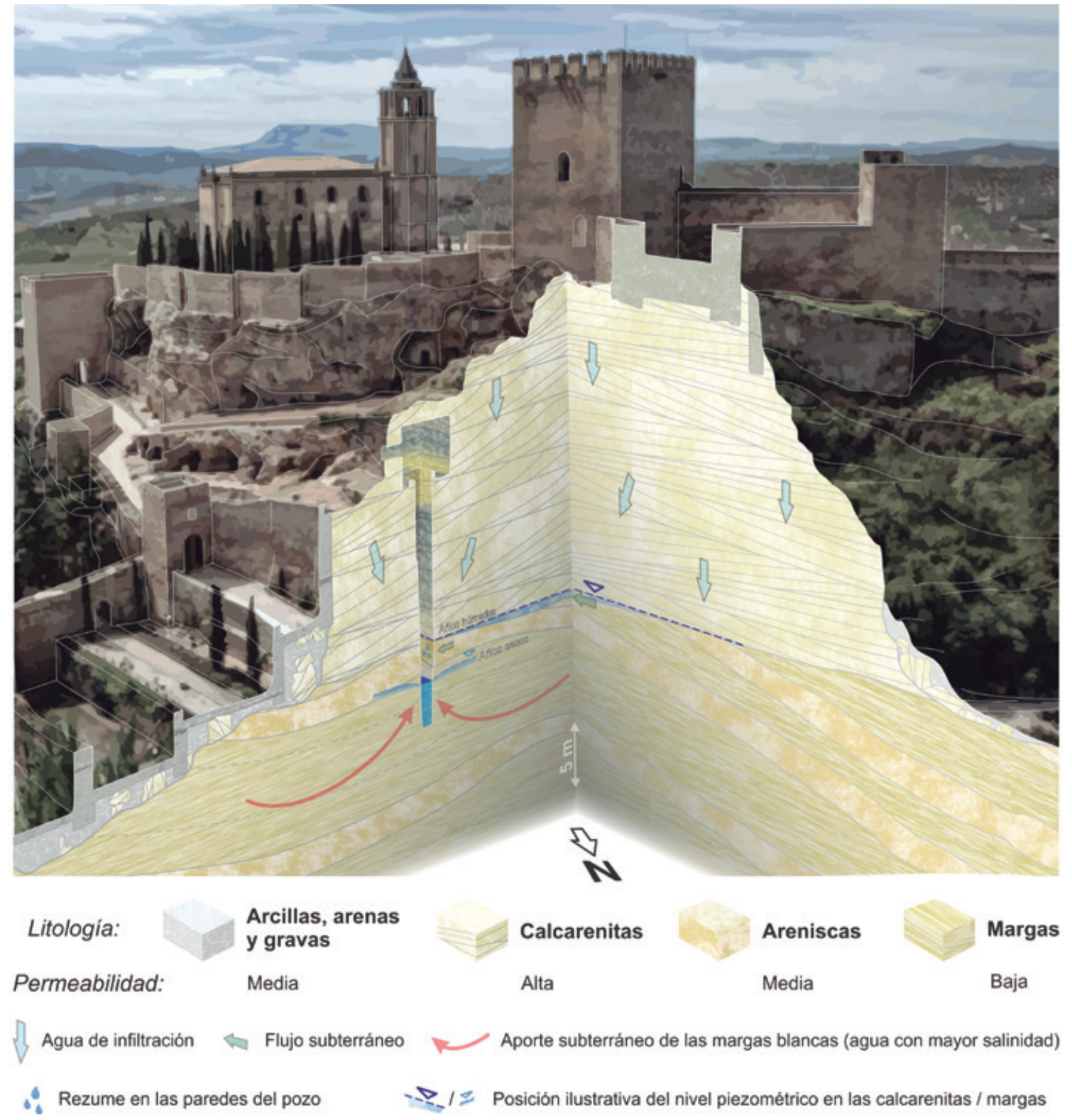

Figura 6. Esquema conceptual de funcionamiento hidrogeológico de las calcarenitas miocenas del cerro de La Mota, en el que está representado el Pozo de la Conquista.

Figure 6. Schematic illustration of the hydrogeological functioning of the calcareous sandstones of Miocene age of the cerro de La Mota, in which the Pozo de la Conquista is represented.

muro del acuífero (Fig. 6). Quedaría por ver cómo respondería la captación bajo un régimen de explotación intensivo, como debió ocurrir durante los periodos de asedio de la Fortaleza.

\section{Conclusiones}

El reducido volumen de recarga y las escasas reservas hídricas del acuífero del cerro de La Mota, por sus particulares condiciones hidrogeológicas, impli- ca que este promontorio de materiales calcareníticos no fuese la fuente de agua ideal para abastecer a los habitantes de la Fortaleza homónima en tiempos de asedio. La facilidad de drenaje de las calcarenitas, por estar colgadas sobre las margas, hace que la explotación del acuífero fuese difícil de ejecutar para captar agua subterránea, pese a las buenas propiedades hidráulicas intrínsecas de estos materiales. La ausencia de un manantial significativo implica que la descarga natural del flujo subterráneo se 
debe producir de forma difusa hacia los materiales de borde, con menor permeabilidad. Además, la calidad del agua subterránea, claramente deteriorada por la litología de los materiales del sustrato y por las actividades antrópicas, supondría un factor limitante adicional para su uso en el abastecimiento de la población. La falta de recursos hídricos subterráneos suficientes y de calidad en este pequeño acuífero explica el gran número de aljibes existentes en la Fortaleza y el paulatino abandono del poblamiento de la misma una vez finalizaron los periodos de conflicto.

Es muy probable que la inutilización del Pozo de la Conquista no fuese la clave definitiva del fin del asedio por parte de las tropas de Alfonso XI; más bien fue el descubrimiento de la galería de aguada que conduce al pozo, lo que facilitó la penetración de las tropas cristianas en el interior de la Fortaleza y su posterior conquista.

Se considera que el gran trabajo de recuperación de todo el conjunto histórico, incluidos pozos, galerías subterráneas de acceso y aljibes, llevado a cabo por las diferentes administraciones debería ser aprovechado por las autoridades para la divulgación científica, además de seguir siendo utilizado como un enclave de especial interés turístico-cultural. En este sentido, se han efectuado trabajos sectoriales de divulgación en diferentes materias (antropología, demografía, agronomía, etc...), además de las meramente históricas. Sin embargo, la explicación didáctica del modelo conceptual de funcionamiento hidrogeológico del acuífero del cerro de La Mota y de su balance hídrico, recogido en el presente trabajo, y la repercusión que en la toma de la antigua alcazaba tuvo la escasa capacidad de regulación natural de las calcarenitas, pueden ser el hilo conductor con el que explicar los aspectos geológicos, hidrológicos y climáticos del entorno donde se asienta la Fortaleza; todos ellos temas científicos menos conocidos.

\section{Agradecimientos}

Este trabajo es una contribución a los grupos de investigación RNM-126 y RNM-308 de la Junta de Andalucía. Se agradece los comentarios constructivos de los dos revisores anónimos.

\section{Referencias}

Azañón, J. M., Roldán, F. J., and Rodríguez Fernández, J. 2012. Fallas y despegues extensionales en el Subbético Central: implicaciones en la evolución Neógena de las
Zonas Externas de la Cordillera Bética. Geogaceta, 52, 13-16.

Calvo Aguilar, C. 2004. El abastecimiento de agua en la Fortaleza de La Mota de Alcalá la Real: Perspectiva arqueológica. In: Funciones de la red castral fronteriza: homenaje a Don Juan Torres Fontes. Alcalá la Real (Jaén). 69-80.

Calvo Aguilar, C. 2017. Las galerías subterráneas de la Fortaleza de La Mota. Ayuntamiento de Alcalá la Real (Jaén). Inédito. 10 pp.

Díaz de Neira, J.A., Enrile Albir, E., Hernaiz Huerta P.P. and López Olmedo, F. 1991. Mapa geológico de España. Serie Magna, 1:50.000, hoja $n^{\circ} 990$ (Alcalá la Real). Instituto Tecnológico Geominero de España, Madrid. 54 pp. ISBN: 84-7840-112-1.

Gollonet, J., González-Ramón, A., Rubio-Campos, J.C., Rosales, M. and Hidalgo-Cano, J.L. 2002. Operaciones de recarga artificial en los acuíferos de Mancha Real-Pegalajar y de Alcalá la Real. Libro homenaje a Manuel del Valle Cardenete. Aportaciones al conocimiento de los acuíferos andaluces. IGME, CHG, Instituto del Agua de Andalucía (COPTJA) y DPG, Madrid, 177-181.

González-Ramón, A., Martín-Montañés, C., Gollonet, J., Martos-Rosillo, S. and Rubio-Campos, J. C. 2009. La recarga artificial como apoyo al abastecimiento de poblaciones. Experiencias en la provincia de Jaén. Boletín Geológico y Minero, 120(2), 289-304.

IGME 1998. Informe de los trabajos previos para la realización de una experiencia de recarga artificial en el acuífero de Los Llanos de Alcalá la Real (Jaén). Instituto Geológico y Minero de España. Informe interno. 87 pp.

Martín-Montañés, C., Rubio-Campos, J.C., Puche-Castilla J., Gay-Torres J.J. and Medina-Vernalte, A. 2018. Contribución al conocimiento hidrogeológico para una gestión sostenible del acuífero de las Calcarenitas de Alcalá la Real-Santa Ana. En: X Simposio del Agua en Andalucía. Unidos por el Agua. Tomo 2-3. (M. Olías, J.C. Cerón, J.J. Durán-Valsero, J.A. López-Geta, C. García de la Noceda, G. Ramos -Editores-), pp 995-1003. Publicaciones del Club del Agua Subterránea, 2-3. ISBN vol. 2: 978-84-09-05454-1. Depósito legal: M-32725-2018.

Martos-Rosillo, S., González-Ramón, A., Jiménez-Gavilán, P., Andreo, B., Durán, J.J. and Mancera, E. 2015. Review on groundwater recharge in carbonate aquifers from SW Mediterranean (Betic Cordillera, S Spain). Environmental Earth Sciences, 74(12), 7571-7581.

Martos-Rosillo, S., Morales, R. and Durán, J.J. 2018. El agua subterránea en la Historia. De cómo la Humanidad se ha abastecido mediante las aguas subterráneas desde el Paleolítico hasta la época de las catedrales Góticas. Colección Planeta Tierra. Ed: Instituto Geológico y Minero de España y los Libros de la Catarata. Madrid. $142 \mathrm{pp}$. 
Mudarra, M., Andreo, B., Barberá, J.A. and Mudry, J. 2014. Hydrochemical dynamics of TOC and $\mathrm{NO}_{3}^{-}$contents as natural tracers of infiltration in karst aquifers. Environmental Earth Sciences, 71(2), 507-523.

Núñez, I., Araguás-Araguás, L., Moreno, L., Rubio, J.C., González-Ramón, A., Pérez-Zabaleta, E., Gollonet, J. 2006. Evolución hidrogeoquímica del acuífero carbonatado profundo de la Loma de Úbeda (Jaén). Boletin Geológico y Minero, 117, 613-616.
Rodríguez Fernández, J. 1982. El Mioceno del sector central de las Cordilleras Béticas. Tesis Doctoral, Universidad de Granada, Granada, 224 pp.

Roldán, F.J., Azañón, J.M. and Rodríguez-Fernández, J. 2012. Desplazamiento extensional del Subbético entre las sierras de Cabra y Alta Coloma (Valdepeñas de Jaén. Zonas Externas de la Cordillera Bética). VIII Congreso Geológico de España. Oviedo. GEOTEMAS, V-13: 484.

Schoeller, H. 1962. Les eaux souterraines. Masson, París, $642 \mathrm{pp}$.

Recibido: julio 2019

Revisado: noviembre 2019

Aceptado: enero 2020

Publicado: marzo 2021 